\title{
BMJ Open Inequalities in physical comorbidity: a longitudinal comparative cohort study of people with severe mental illness in the UK
}

\author{
Siobhan Reilly, ${ }^{1}$ Ivan Olier, ${ }^{2}$ Claire Planner, ${ }^{3}$ Tim Doran, ${ }^{4}$ David Reeves, ${ }^{5}$ \\ Darren M Ashcroft, ${ }^{6}$ Linda Gask, ${ }^{3}$ Evangelos Kontopantelis ${ }^{3,7}$
}

To cite: Reilly S, Olier I, Planner $\mathrm{C}$, et al. Inequalities in physical comorbidity: a longitudinal comparative cohort study of people with severe mental illness in the UK. BMJ Open 2015;5: e009010. doi:10.1136/ bmjopen-2015-009010

- Prepublication history and additional material is available. To view please visit the journal (http://dx.doi.org/ 10.1136/bmjopen-2015009010).

The study was carried out in the University of Manchester's Institute of Population Health and Lancaster University's Division of Health Research.

Received 11 June 2015 Revised 14 August 2015 Accepted 4 September 2015

CrossMark

For numbered affiliations see end of article.

Correspondence to

Dr Siobhan Reilly;

s.reilly@lancaster.ac.uk

\section{ABSTRACT}

Objectives: Little is known about the prevalence of comorbidity rates in people with severe mental illness (SMI) in UK primary care. We calculated the prevalence of SMI by UK country, English region and deprivation quintile, antipsychotic and antidepressant medication prescription rates for people with SMI, and prevalence rates of common comorbidities in people with SMI compared with people without SMI.

Design: Retrospective cohort study from 2000 to 2012.

Setting: 627 general practices contributing to the Clinical Practice Research Datalink, a UK primary care database.

Participants: Each identified case (346 551) was matched for age, sex and general practice with 5 randomly selected control cases (1 732755$)$ with no diagnosis of SMI in each yearly time point.

Outcome measures: Prevalence rates were calculated for 16 conditions.

Results: SMI rates were highest in Scotland and in more deprived areas. Rates increased in England, Wales and Northern Ireland over time, with the largest increase in Northern Ireland $(0.48 \%$ in $2000 / 2001$ to $0.69 \%$ in $2011 / 2012$ ). Annual prevalence rates of all conditions were higher in people with SMI compared with those without SMI. The discrepancy between the prevalence of those with and without SMI increased over time for most conditions. A greater increase in the mean number of additional conditions was observed in the SMI population over the study period (0.6 in 2000/ 2001 to 1.0 in 2011/2012) compared with those without SMI (0.5 in 2000/2001 to 0.6 in 2011/2012). For both groups, most conditions were more prevalent in more deprived areas, whereas for the SMI group conditions such as hypothyroidism, chronic kidney disease and cancer were more prevalent in more affluent areas.

Conclusions: Our findings highlight the health inequalities faced by people with SMI. The provision of appropriate timely health prevention, promotion and monitoring activities to reduce these health inequalities are needed, especially in deprived areas.

\section{Strengths and limitations of this study}

- Large UK longitudinal study to explore the prevalence of severe mental illness (SMI) and comorbidity in the context of deprivation covering 12 years (2000-2012).

- Differences in the prevalence of SMI over time, between countries in the UK and regions in England are explored; increases observed between 2000 and 2012 and highest in areas of high deprivation.

- This research highlights the rising inequalities in the pattern and number of different comorbidities of this group of patients, a variable pattern of comorbidity across the different SMI subgroups and areas of high and low deprivation and has the potential to inform the provision of appropriate and timely health prevention, promotion and monitoring activities.

- Routinely collected clinical data were used, so we were not able to match controls on other important parameters such as obesity, unemployment, ethnicity, smoking status, alcohol or illegal drug use which may not be recorded.

\section{INTRODUCTION}

It is well established that the physical health of people with severe mental illness (SMI) is much poorer than for people without SMI and that the causes of poor physical health in people with a SMI are complex and interactive. ${ }^{1}$ The factors that account for this include adverse effects of antipsychotic medication $^{2}$ and unhealthy lifestyle behaviours which increase the likelihood of developing obesity, hypercholesterolaemia and metabolic syndrome, which in turn increase the risk of chronic diseases such as diabetes mellitus. Other barriers to recognising and managing physical conditions include difficulty in understanding healthcare advice, reduced 
motivation to adopt new lifestyle changes, poor treatment compliance, cognitive deficits, reduced pain sensitivity (induced by antipsychotic medication), poor communication and social skills. ${ }^{3}{ }^{4}$ Higher risk of comorbidity is often compounded by problems of engagement with the National Health Service (NHS) healthcare system, for example, reluctance of general practitioners (GPs) to participate in care, ${ }^{5}{ }^{6}$ which is reflected in the likelihood of patients being opted out (appropriately or not) but also refusing treatment. ${ }^{5} 78$ There is some evidence that health prevention and promotion activities in primary care are less frequent for people with SMI despite frequent contact with the system. ${ }^{9}$ As a result, premature mortality is much higher in people with SMI compared with those without SMI. ${ }^{10}$ A number of both system and individual actions are necessary to address gaps in the treatment of physical health in people with SMI. ${ }^{4}$

Several cross-sectional studies have indicated that individuals with SMI have increased rates of physical illness compared with the general population: ${ }^{2}{ }^{12-16}$ however, these studies refer to data for limited periods of time, focus on Scotland or London. We extend this work by describing both SMI rates and patterns of comorbidity in a matched UK population over a 12-year period. A better understanding of comorbidity for people with SMI is necessary for improving estimates to inform policy and planning services. ${ }^{17}$ This paper examines the:

1. Prevalence of SMI in the UK by country (England, Northern Ireland (NI), Scotland and Wales), each region in England and each deprivation quintile in the UK, during the period 2000-2012.

2. Prevalence rates of 16 comorbidities in people with SMI during (1) 2000-2012 and (2) 2011/2012 for different types of SMI diagnoses (schizophrenia, bipolar disorder, affective disorder and other types of psychosis), compared with people without SMI and by the most affluent and most deprived quintile and (3) the 5-year period 2007/2008-2011/2012 for the combinations of comorbidities, for both people with and without SMI.

\section{METHODS}

The Clinical Practice Research Datalink (CPRD) is a large primary care database of anonymised longitudinal medical records which contains detailed information on diagnoses, referrals, prescribed treatments and test results. The version we analysed has been described in detail elsewhere. ${ }^{18}$ We used all available data from 627 practices to extract diagnoses information and aggregated it in 12 yearly bins, from 1 April 2000 to 31 March 2012.

Generating a code list for SMI and other conditions We used Read codes to identify the presence of SMI. First, we identified relevant keywords (or key-stubs) and codes, for example, 'paranoi' and E1*, covering the mental health domain (see online appendix table A1). Next, the CPRD was searched for codes that matched the list in either the code or the description field. Finally, the matched code list was reviewed by clinical experts, and a final conservative list of codes was agreed. SMI was defined as: schizophrenia, affective disorder (divided into bipolar or unspecified affective disorder) or other types of psychoses, consistent with the inclusion criteria for SMI registers in primary care general practice in the UK as part of the Quality and Outcomes Framework (QOF) financial incentive scheme. The QOF was introduced in 2004 and links GP's pay with achievement of targets set across a range of chronic conditions. $^{19}$

The research team selected 16 conditions from an extended list, based on previous work, incentivisation under the QOF and a review of existing literature. The QOF is relevant since quality of recording is excellent for its domains and we included all except depression (because of coding complexities and potential overlap with SMI) and obesity. The conditions were: hypertension, diabetes (type I and II), asthma, hypothyroidism, osteoarthritis, chronic kidney disease (CKD), learning disability, coronary heart disease, epilepsy, chronic obstructive pulmonary disease (COPD), cancer, stroke, heart failure, rheumatoid arthritis, dementia and psoriasis. These were discussed and agreed a priori by all authors, and codes associated with these conditions were obtained through a similar approach to the one used for patients with SMI and were mainly developed for previous work..$^{20}$ All the code lists we used, as well as the SMI categorisation, are available from http://www. clinicalcodes.org, ${ }^{21}$ while more details on the Read code selection process in this SMI context have been provided elsewhere. ${ }^{22}$

Within each year, all patients registered with a CPRD practice for the whole year and aged 18 or over were eligible for inclusion. The final SMI Read code list was used to identify cases with schizophrenia, affective psychoses (bipolar disorder or other unspecified affective psychosis) and other types of psychosis, in line with the diagnoses used when compiling primary care QOF SMI registers. ${ }^{23}$ If an individual received more than one SMI diagnosis over the study period, we used the last available diagnosis to retrospectively 'correct' the original diagnosis (ie, we assumed that the latest diagnosis was the correct one). Within each year, each SMI case was then matched on age, sex and general practice to five randomly selected patients not associated with SMI up until that time point.

\section{Other sociodemographic characteristics}

Deprivation was measured using the 2007 Index of Multiple Deprivation (IMD) score in England, ${ }^{24}$ applied to the practice postcode. Analogous deprivation indexes were used for Welsh, Scottish and NI practices. One of five deprivation quintiles, based on the deprivation 
distribution within each country, was assigned to each individual in the sample.

\section{Medications}

We reported the number of individuals with one or more prescriptions within a year for each of the following medications: antipsychotic drugs (first, second generation and depot injections), tricyclic antidepressants (TCAs), selective serotonin reuptake inhibitors (SSRIs) and other antidepressants.

\section{Analysis}

SMI prevalence rates were calculated overall, by deprivation quintile and by practice region, across the study period. We report patient characteristics for both SMI and control cases, including prevalence rates for the investigated comorbidities. Finally, we created detailed comorbidity mapping tables for both groups, a common practice when investigating disease clusters, ${ }^{12}{ }^{25}$ to identify relevant patterns of comorbidity in SMI cases and investigate how they differed to what is observed for controls. Prevalence rates were calculated annually over the study period (2000/2001-2011/2012), although we focused on the last financial year to investigate if the comorbidity patterns differed by SMI diagnosis type (schizophrenia, bipolar disorder, affective disorder and other types of psychosis). We also calculated comorbidity maps for combinations of comorbidities, for both SMI and controls, aggregated over 5 years (2007/2008-2011/ 2012).

\section{RESULTS}

The number of practices included in the sample ranged from 434 practices in 2000/2001 to 569 in 2006/2007 (table 1; online appendix figure A1). Numbers of individuals with SMI rose year on year from 19658 in 2000/2001 (434 practices) to a high of 33117 in 2009/2010 (556 practices) and declined in subsequent years (table 1 ).

In Scotland, in 2011/2012, the annual prevalence of SMI was $0.73 \%$ compared with $0.69 \%$ in NI, $0.65 \%$ in Wales and $0.63 \%$ in England (table 2 and figure 1). The prevalence rate of SMI in Scotland did not increase overall during the study period, though in the most deprived quintile, some increases were observed. In contrast, rates increased in England, Wales and especially in NI (from 0.48 in 2000/2001 to 0.69 in 2011/2012). With the exception of the South West, the annual prevalence rate of SMI increased across all English regions over the study period (table 2). The greatest increases were observed in the North East, North West and the East Midlands. SMI prevalence was the highest in areas in the two highest deprivation quintiles (table 2 and figure 1 ). Changes over time were more evident in the most deprived quintile in NI with rates more than doubling over the 12-year period ( 0.49 in $2000 / 2001$ to 1.16 in $2011 / 2012$ ), with a great rise in $2007 / 2008$ (table 2).
The mean number of years since the first SMI diagnosis increased from 11.7 (SD 11.5) in 2000/2001 to 13.2 (SD 11.8 ) in $2011 / 2012$ and the mean years since the final diagnosis increased from 9.1 (SD 11.3) in 2000/2001 to 11.5 (SD 11.0) in 2011/2012 (table 3).

The prevalence rates for all of the diagnostic categories (bipolar disorder: 0.13-0.19; other SMI: 0.18-0.23; and schizophrenia: 0.11-0.14) increased over the study period with the least increase in unspecified/other affective psychosis (0.06-0.07).

\section{Medication: antipsychotic medication and antidepressants}

The number of individuals with one or more prescription for first generation antipsychotic medications has steadily declined over the 12 years (from $20.26 \%$ to $9.78 \%$ ), whereas second generation antipsychotic medications have steadily increased (from $18.1 \%$ to $43 \%$ ). Decreases in depot injections were observed over time as shown in table 3. An increase over the time period was observed for SSRIs (from $18.1 \%$ to $25.75 \%$ ), whereas a decrease was observed for TCAs (from $14.03 \%$ to $9.21 \%$ ).

\section{Prevalence rates of comorbidities}

Annual prevalence rates varied over the 12-year period for those with and without SMI (table 1). There appeared to be a greater increase in the mean number of additional conditions in the SMI population over the study period (0.6 in $2000 / 2001$ to 1.0 in $2011 / 2012$ ) compared with control cases $(0.5$ in $2000 / 2001$ to 0.6 in 2011/2012; table 1).

Over time, prevalence rates for comorbidities generally increased for both groups but the increases were greater for the SMI group. For example, hypertension prevalence increased from 12.2 to 18.3 between $2000 /$ 2001 and 2011/2012 for the SMI group, but for the control group, the increase was smaller from 14.2 to 16.3 over the same time period. There were some notable exceptions: the rates for coronary heart disease and heart failure fell for both groups, and the rates for stroke remained relatively stable for the SMI group but fell for the controls.

Although percentage of patients with two specific conditions differed, comorbidity combinations were similar for the patients with SMI and matched controls. The most common comorbidity combination (percentage of patients with 2 conditions) was diabetes and hypertension and was experienced by $4.4 \%$ of patients with SMI (figure 2; online appendix tables A2 and A3). The percentages of people with one of the conditions who also have other conditions were higher for the SMI group for most conditions (see online appendix table A4 and figure A2). However, higher rates of hypertension were observed in the controls with a diagnosis of diabetes, asthma, hypothyroidism, osteoarthritis, CKD, COPD and heart failure.

A variable pattern of comorbidity was observed across the different SMI subgroups and areas of high and low deprivation (see online appendix tables A5-7). When we focused on 2011/2012 data and compared SMI and 
Table 1 Characteristics of the SMI population and the matched controls, over time

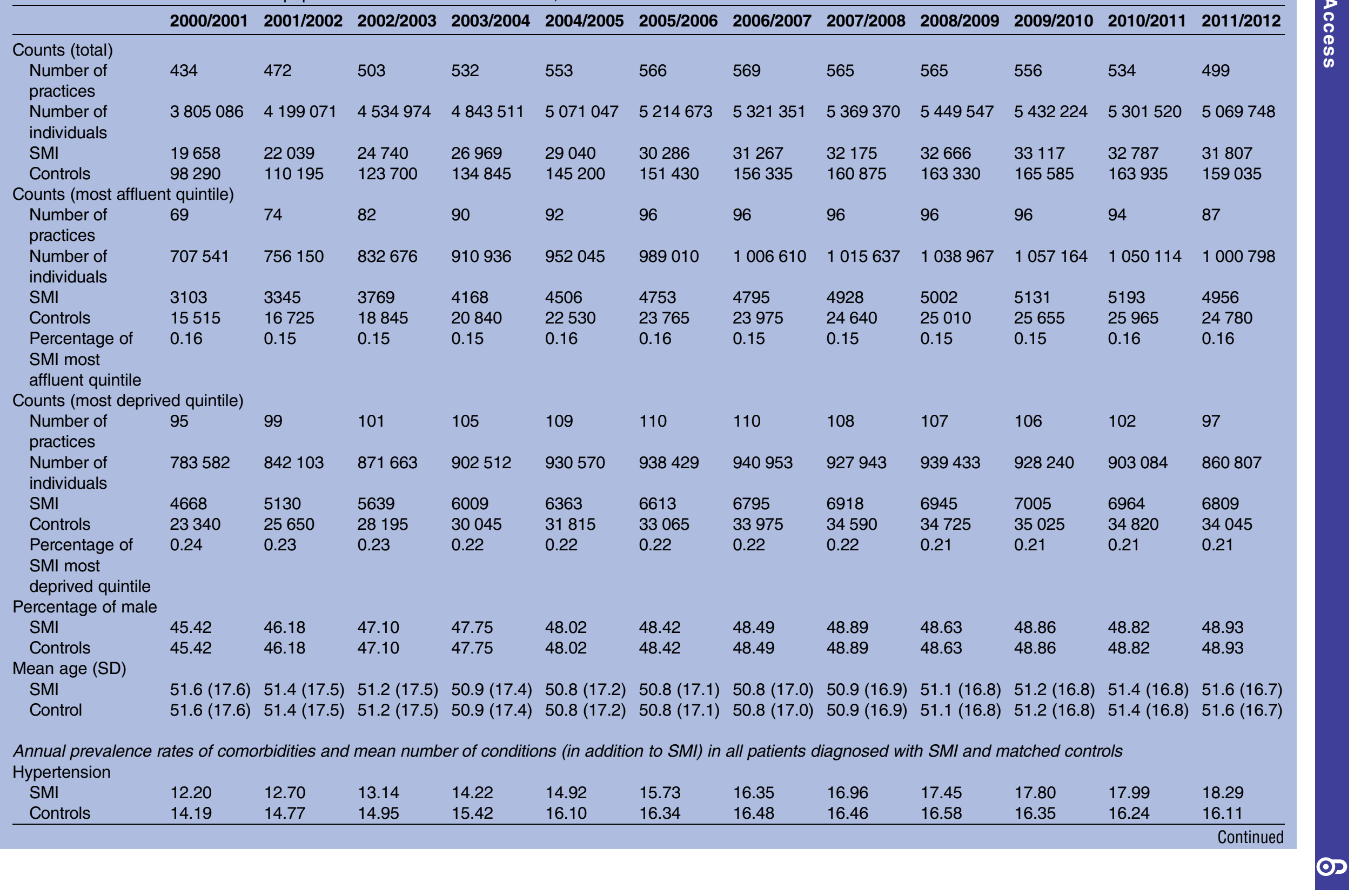


Table 1 Continued

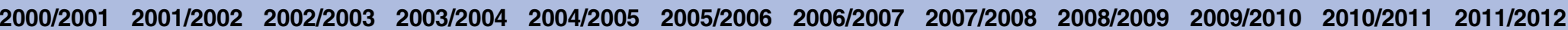

\begin{tabular}{|c|c|c|c|c|c|c|c|c|c|c|c|c|}
\hline \multicolumn{13}{|c|}{ Diabetes (type I and II) } \\
\hline SMI & 5.29 & 5.79 & 6.07 & 6.48 & 7.18 & 7.72 & 8.34 & 8.79 & 9.33 & 9.82 & 10.41 & 11.11 \\
\hline Controls & 3.53 & 3.77 & 3.85 & 4.14 & 4.33 & 4.49 & 4.70 & 4.90 & 5.05 & 5.16 & 5.36 & 5.55 \\
\hline \multicolumn{13}{|l|}{ Asthma } \\
\hline SMI & 5.80 & 6.14 & 6.48 & 7.06 & 7.29 & 7.54 & 7.67 & 7.82 & 8.20 & 8.29 & 8.18 & 7.88 \\
\hline Controls & 5.44 & 5.48 & 5.74 & 5.75 & 5.93 & 5.98 & 5.88 & 6.01 & 5.98 & 6.03 & 5.85 & 5.47 \\
\hline \multicolumn{13}{|c|}{ Hypothyroidism } \\
\hline SMI & 5.51 & 5.75 & 6.23 & 6.84 & 7.45 & 8.05 & 8.32 & 8.56 & 8.87 & 9.00 & 9.06 & 9.18 \\
\hline Controls & 2.96 & 3.20 & 3.19 & 3.54 & 3.78 & 3.96 & 4.01 & 4.05 & 4.14 & 4.25 & 4.19 & 4.24 \\
\hline \multicolumn{13}{|c|}{ Osteoarthritis } \\
\hline SMI & 8.98 & 9.05 & 9.21 & 9.44 & 9.60 & 9.80 & 9.80 & 10.05 & 10.41 & 10.38 & 10.71 & 10.79 \\
\hline Controls & 9.36 & 9.42 & 9.20 & 9.24 & 9.28 & 9.51 & 9.53 & 9.44 & 9.58 & 9.61 & 9.63 & 9.50 \\
\hline \multicolumn{13}{|c|}{ Chronic kidney disease } \\
\hline SMI & 0.28 & 0.32 & 0.45 & 0.55 & 0.67 & 0.96 & 5.48 & 7.02 & 7.53 & 7.88 & 8.01 & 8.24 \\
\hline Controls & 0.26 & 0.29 & 0.30 & 0.36 & 0.44 & 0.57 & 3.19 & 3.89 & 4.14 & 4.27 & 4.25 & 4.16 \\
\hline \multicolumn{13}{|c|}{ Learning disability } \\
\hline SMI & 1.33 & 1.31 & 1.28 & 1.24 & 1.29 & 1.37 & 1.48 & 1.46 & 1.46 & 1.75 & 1.89 & 1.86 \\
\hline Controls & 0.13 & 0.16 & 0.15 & 0.15 & 0.15 & 0.16 & 0.18 & 0.18 & 0.16 & 0.22 & 0.21 & 0.22 \\
\hline \multicolumn{13}{|c|}{ Coronary heart disease } \\
\hline SMI & 5.71 & 5.47 & 5.44 & 5.21 & 4.97 & 4.82 & 4.76 & 4.63 & 4.51 & 4.50 & 4.47 & 4.51 \\
\hline Controls & 5.61 & 5.56 & 5.31 & 4.97 & 4.81 & 4.60 & 4.43 & 4.24 & 4.00 & 3.85 & 3.66 & 3.60 \\
\hline \multicolumn{13}{|l|}{ Epilepsy } \\
\hline SMI & 2.03 & 2.19 & 2.17 & 2.40 & 2.54 & 2.60 & 2.56 & 2.61 & 2.55 & 2.59 & 2.66 & 2.58 \\
\hline Controls & 0.70 & 0.76 & 0.75 & 0.72 & 0.82 & 0.75 & 0.77 & 0.75 & 0.76 & 0.70 & 0.71 & 0.70 \\
\hline \multicolumn{13}{|l|}{ COPD } \\
\hline SMI & 1.89 & 2.01 & 2.05 & 1.94 & 2.10 & 2.26 & 2.49 & 2.74 & 2.94 & 3.12 & 3.20 & 3.46 \\
\hline Controls & 1.36 & 1.40 & 1.47 & 1.43 & 1.56 & 1.60 & 1.60 & 1.66 & 1.72 & 1.72 & 1.81 & 1.80 \\
\hline \multicolumn{13}{|l|}{ Cancer } \\
\hline SMI & 2.76 & 2.77 & 2.93 & 3.07 & 3.12 & 3.22 & 3.38 & 3.46 & 3.50 & 3.65 & 3.83 & 4.11 \\
\hline Controls & 2.70 & 2.78 & 2.74 & 2.81 & 2.87 & 2.96 & 3.09 & 3.06 & 3.14 & 3.21 & 3.39 & 3.44 \\
\hline \multicolumn{13}{|l|}{ Stroke } \\
\hline SMI & 3.64 & 3.69 & 3.63 & 3.53 & 3.47 & 3.52 & 3.45 & 3.55 & 3.52 & 3.59 & 3.75 & 3.75 \\
\hline Controls & 2.51 & 2.48 & 2.42 & 2.24 & 2.21 & 2.09 & 2.15 & 2.08 & 2.09 & 1.98 & 1.97 & 2.07 \\
\hline \multicolumn{13}{|l|}{ Heart failure } \\
\hline SMI & 2.28 & 2.05 & 2.07 & 1.99 & 1.86 & 1.74 & 1.55 & 1.44 & 1.34 & 1.31 & 1.37 & 1.41 \\
\hline Controls & 1.64 & 1.55 & 1.44 & 1.30 & 1.15 & 1.06 & 0.97 & 0.90 & 0.88 & 0.79 & 0.77 & 0.79 \\
\hline \multicolumn{13}{|c|}{ Rheumatoid arthritis } \\
\hline SMI & 0.92 & 0.89 & 0.84 & 0.86 & 0.91 & 0.88 & 0.89 & 0.92 & 0.90 & 0.95 & 0.99 & 1.04 \\
\hline Controls & 0.91 & 0.97 & 0.94 & 0.99 & 0.95 & 0.95 & 0.97 & 0.98 & 0.92 & 0.91 & 0.97 & 0.95 \\
\hline
\end{tabular}




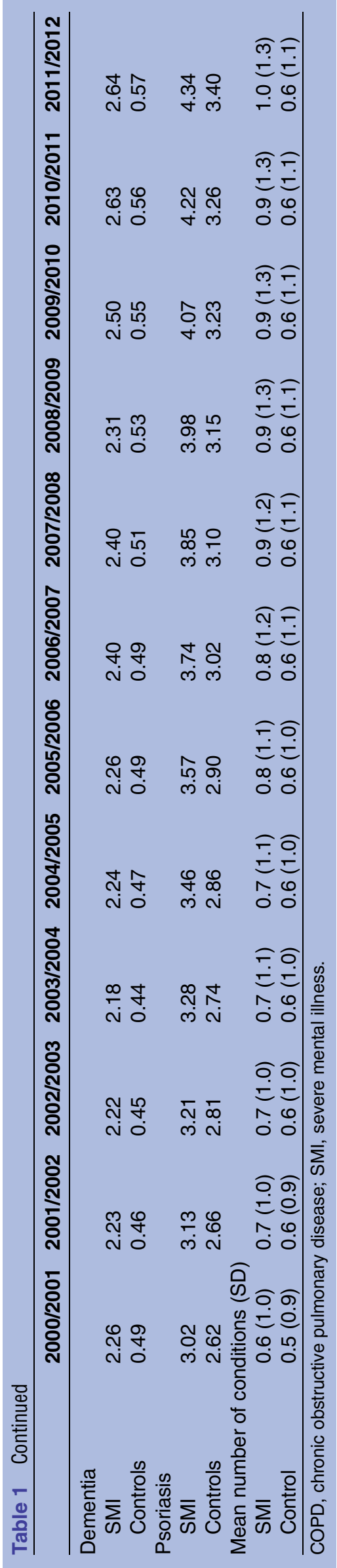

control cases within each SMI diagnosis subgroup, we observed differences in prevalence rates for almost all conditions in all groups (see online appendix table A5). We noted large differences between the prevalence rates of a number of conditions such as diabetes mellitus, hypothyroidism, CKD for people with SMI and those without SMI. There were also differences between SMI diagnoses groups (schizophrenia, bipolar disorder, affective disorder and other types of psychosis). For example, the difference between prevalence rates of people with schizophrenia and controls who also had a diabetes diagnosis (13.42 compared with 6.01) was larger than the difference between the other three SMI diagnoses groups and controls. The difference between prevalence rates of people with bipolar disorder and controls who also had a hypothyroidism diagnosis (12.77 compared with 4.58) and a CKD diagnosis (10.41 compared with 3.9) than the difference between the other three SMI diagnoses groups and controls. In addition, for diabetes mellitus, asthma, CHD, COPD, learning disability, osteoarthritis and epilepsy, we observed higher prevalence rates in people with SMI in the most deprived quintile (see online appendix table A6). For hypothyroidism, CKD, psoriasis, cancer, stroke and dementia, prevalence rates were higher in the most affluent quintile (see online appendix table A7).

\section{DISCUSSION}

\section{Findings}

This is the first large longitudinal study to explore the prevalence of SMI and comorbidity in the context of deprivation. We identified a number of key findings: First, we found that the prevalence of SMI, in the UK, increased over the 12-year period from 2000 to 2012. The increase was consistent across all diagnosis subgroups but highest in bipolar disorder and other SMI. Increases were highest in areas of high social deprivation. Second, the difference in rates across England, NI, Wales and Scotland has narrowed overtime. Third, the age at which people received their diagnosis lowered over the 12 years (age stayed constant while the mean number of years since the first SMI diagnosis increased). Fourth, we observed an increase in the average number of reported comorbidities for the SMI group. Some conditions increased at a higher rate for those with a SMI diagnosis: COPD, diabetes, hypothyroidism and asthma. Whereas the rates for hypertension, CKD and stroke increased in the SMI group in contrast to control cases which showed a decrease. This also coincides with yearly increase in the proportion of people with SMI with one or more prescription of atypical antipsychotic medications. Finally, a variable pattern of comorbidity was observed across the different SMI subgroups and areas of high and low deprivation.

\section{Comparison with previous research}

Our study is one of the latest in a growing number of studies that have used electronic patient health records 
Table 2 Annual severe mental illness (SMI) prevalence rates (all patients diagnosed with SMI) by geographical location and area deprivation quintile, over time

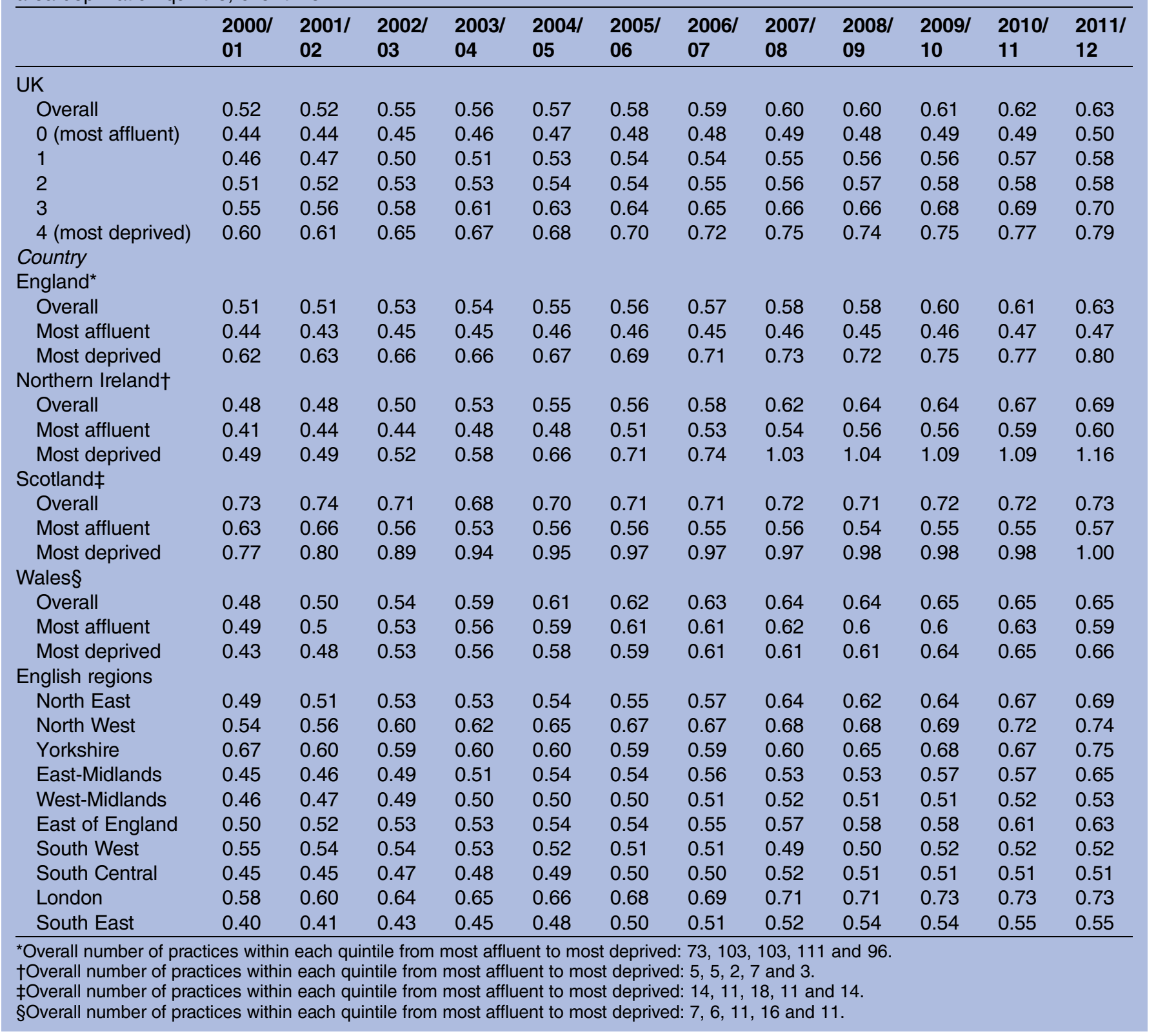

to examine epidemiological data and multimorbidity associated with people with SMI. ${ }^{2} 131526$ The prevalence rate of SMI observed in the final study year $(2011 / 2012)$ is lower than reported by the English QOF (0.63 compared with 0.87), ${ }^{18}$ and previous estimates. ${ }^{27}$ Our 2007 rates for Scotland $(0.71 / 0.72)$ were similar to those recently reported $(0.70) .{ }^{13}$ Differences are common in studies which use different Read code lists to define SMI. For example, the lower rates in our study may be partly attributed to the exclusion of $\mathrm{drug} / \mathrm{alcohol}$-induced psychoses, organic psychoses, dementia, unipolar depression, personality disorders and psychotic disorders in childhood/adolescence. The publication of our Read code lists, through http://www.clinicalcodes.org, will facilitate future research in this field and the comparison of rates across future studies.
It is not possible within this study to explain the reasons for the increase in SMI rates over time. Our results indicate that people are getting diagnosed earlier. However, further research is needed to explore the factors that may contribute to these observed increases.

Although there have been recent studies providing estimates of prevalence, they examined a more limited set of comorbidities, ${ }^{2} 16$ they did not have UK coverage or provide estimates for all SMI subgroups. ${ }^{13} 15$ To our knowledge, there are currently no other comparable studies that have examined rates of comorbidities over such a long period of time. Some of our annual findings are consistent with other studies. The 2011/2012 prevalence rates for eight conditions (hypertension, asthma, hypothyroidism, CKD, epilepsy, cancer, stroke, psoriasis) were higher than reported in two previous studies. ${ }^{13} 15$ 

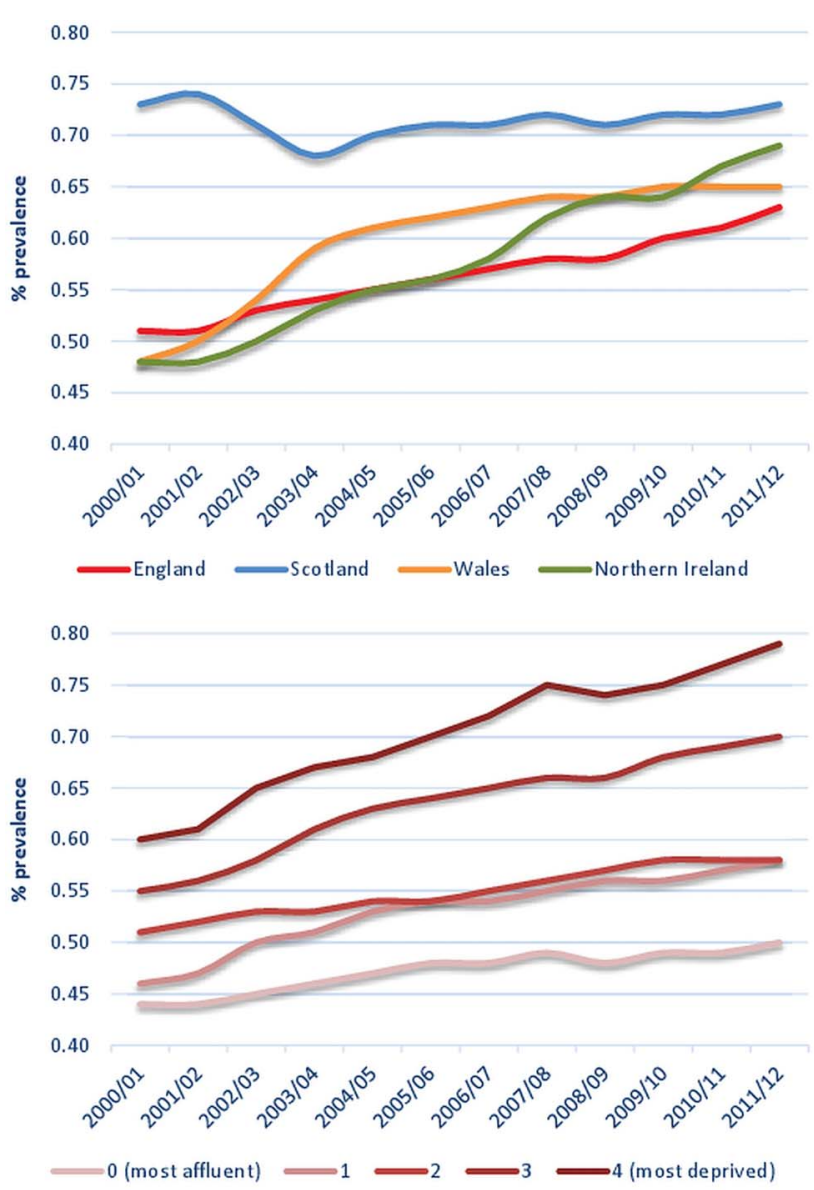

Figure 1 Prevalence of severe mental illness by UK country (top) and deprivation quintile (bottom), over time.

Other UK studies have not reported dementia prevalence rates for individuals with SMI; our 2011/2012 estimates were higher than the general population (2.64 compared with 0.57 ). For the same time period, Barnett et $a l^{12}$ reported 0.7 with dementia in the general population comparing to 0.49 in our control population for the same year (2007). When we compared our prevalence rates for the controls with this study for comparable conditions and year, most rates were within a $\pm 0.5 \%$ range. The rates in our study were higher for diabetes (16.48 compared with 13.4), CKD (3.19 compared with 1.9), psoriasis (3.02 compared with 0.7) but lower for COPD (1.6 compared with 3.2). Although there is a growing literature on analysis of clustering conditions, ${ }^{25}$ it was not possible to compare our findings on the most common comorbidity combinations (percentage of patients with 2 conditions) with any of the SMI studies. Although one other study examined combinations of comorbidities, percentages were not reported. ${ }^{15}$

Others have also shown that the presence of a mental health disorder (not just SMI) increased as the number of physical morbidities and is much more likely in more deprived people. ${ }^{12}$ It is possible that the increased number of certain conditions may be linked with increased prescriptions of antipsychotic medications. ${ }^{28-30}$ Furthermore, the lower annual prevalence levels in early years in some conditions may be due to under-reporting in primary care. It is possible that initiatives such as the QOF, the Commissioning for Quality and Innovation (CQUINs) payments framework and the Cardiometabolic Health Resource ${ }^{31}$ may be helping to improve diagnosis and thus leading to increases in prevalence rates. This may indicate a general underdiagnosis of conditions and may therefore be an underestimation of the prevalence among patients with SMI. Others have noted that studies based on medical records will underestimate multimorbidity because some diseases are undiagnosed, and because they will not identify people who do not consult. ${ }^{32}$ In a separate paper, we show that consultations increased over the 12-year period, ${ }^{33}$ which fits with previous evidence indicating that people who consult more often may have more conditions diagnosed. ${ }^{34}$ Smith et al observed a systematic underrecognition which might contribute to the substantial cardiovascular-related morbidity and premature mortality observed in patients with schizophrenia. ${ }^{12}$ Our data would suggest that this may also be the case for other SMI subgroups too.

The provision of good medical care tends to vary inversely with need, ${ }^{35}$ and could account for the higher rates of SMI and comorbidity in areas of higher social deprivation; however, we speculate that better case finding, driven by the QOF, partially accounts for the observed increases in the comorbidity burden for the QOF-incentivised SMI group, not observed in the matched controls. ${ }^{2036}$

Further research is required to examine the upward trend in many of the conditions and action is urgently required to identify the accurate prevalence rates for people with SMI. The recent recommendation to include mental health experts on all National Institute for Health and Care Excellence (NICE) guideline development groups for physical conditions to ensure that the mental health aspects of conditions are comprehensively considered is timely. ${ }^{37}$ The findings are relevant for NHS strategic and operational plans addressing outcomes related to improving health, reducing health inequalities and parity of esteem. ${ }^{38}$ There is an ambition to achieve a genuine parity of esteem between mental and physical health by 2020, and an expectation that each clinical commissioning group is spending on mental health services in 2015/2016 increases in real terms. ${ }^{39}$ The patterns of comorbidities in the most affluent and most deprived quintiles indicate that the effect of deprivation is worth further exploration in conjunction with the impact on clinical activity. For example, our data may suggest that people with SMI registered at a more affluent practice may be more likely to undergo regular monitoring making the diagnosis of unrelated diseases such as cancer and hypothyroidism more likely. It may also be worth investigating comorbidity interactions as the diagnosis of 
Table 3 Prevalence, number and gender for all individuals with severe mental illness (SMI) diagnosis*, mean years with first and latest SMI diagnosis and prescribed medicationst, over time

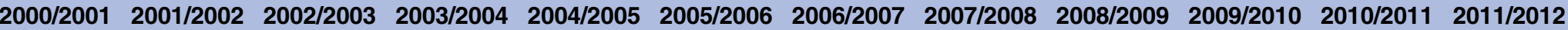

\begin{tabular}{|c|c|c|c|c|c|c|c|c|c|c|c|c|}
\hline \multicolumn{13}{|c|}{ Prevalence of individuals with SMI diagnosis } \\
\hline Schizophrenia & 0.11 & 0.11 & 0.12 & 0.12 & 0.13 & 0.13 & 0.13 & 0.14 & 0.13 & 0.14 & 0.14 & 0.14 \\
\hline Bipolar disorder & 0.13 & 0.13 & 0.14 & 0.15 & 0.15 & 0.16 & 0.16 & 0.17 & 0.17 & 0.18 & 0.18 & 0.19 \\
\hline $\begin{array}{l}\text { Unspecified/other } \\
\text { affective psychosis }\end{array}$ & 0.06 & 0.06 & 0.06 & 0.06 & 0.06 & 0.06 & 0.06 & 0.07 & 0.07 & 0.07 & 0.07 & 0.07 \\
\hline $\begin{array}{l}\text { Other types of } \\
\text { psychosis }\end{array}$ & 0.18 & 0.18 & 0.19 & 0.2 & 0.2 & 0.2 & 0.2 & 0.21 & 0.21 & 0.21 & 0.22 & 0.23 \\
\hline \multicolumn{13}{|c|}{ Number of individuals with SMI diagnosis (not exclusively) } \\
\hline Schizophrenia & 4098 & 4674 & 5268 & 5842 & 6339 & 6727 & 7059 & 7281 & 7296 & 7323 & 7205 & 7029 \\
\hline Bipolar disorder & 4906 & 5613 & 6352 & 7083 & 7784 & 8259 & 8701 & 9055 & 9332 & 9715 & 9727 & 9496 \\
\hline $\begin{array}{l}\text { Unspecified/other } \\
\text { affective psychosis }\end{array}$ & 1938 & 2150 & 2392 & 2573 & 2839 & 2999 & 3255 & 3398 & 3551 & 3690 & 3696 & 3605 \\
\hline Other SMI & 6862 & 7691 & 8764 & 9551 & 10226 & 10628 & 10857 & 11226 & 11484 & 11623 & 11665 & 11631 \\
\hline \multicolumn{13}{|c|}{ Percentage of male individuals with SMI diagnoses } \\
\hline Schizophrenia & 54.86 & 55.63 & 57.52 & 58.37 & 58.98 & 59.86 & 60.41 & 60.86 & 60.68 & 61.35 & 61.5 & 61.79 \\
\hline Bipolar disorder & 39.52 & 39.66 & 39.97 & 39.93 & 39.92 & 39.77 & 39.46 & 39.72 & 39.71 & 39.66 & 39.8 & 39.49 \\
\hline $\begin{array}{l}\text { Unspecified/other } \\
\text { affective psychosis }\end{array}$ & 32.04 & 33.26 & 34.36 & 35.29 & 35.51 & 35.45 & 36.65 & 37.7 & 37.37 & 37.32 & 37.28 & 37.14 \\
\hline Other types of & 48.32 & 49.25 & 49.55 & 50.38 & 50.76 & 51.4 & 51.39 & 51.88 & 51.68 & 52.34 & 52.2 & 52.51 \\
\hline
\end{tabular}

psychosis

Mean number of years with SMI diagnosis (SD)

First SMI diagnosis $11.7(11.5) \quad 11.8(11.6) \quad 11.8(11.6) \quad 11.8(11.6) \quad 11.8(11.6) \quad 12.1(11.7) \quad 12.2(11.7) \quad 12.3(11.6) \quad 12.6(11.7) \quad 12.8(11.7) \quad 13.0(11.7) \quad 13.2(11.8)$

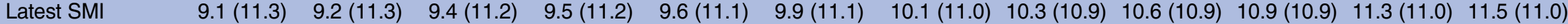
diagnosis

Antipsychoticsł

\begin{tabular}{|c|c|c|c|c|c|c|c|c|c|c|c|c|}
\hline $\begin{array}{l}\text { First generation } \\
\text { (conventional) }\end{array}$ & 20.26 & 20.11 & 17.64 & 15.31 & 14.79 & 13.84 & 12.71 & 11.94 & 11.03 & 10.12 & 9.39 & 8.78 \\
\hline $\begin{array}{l}\text { Second generation } \\
\text { (atypical) }\end{array}$ & 18.1 & 23.03 & 27.12 & 30.68 & 33.33 & 35.77 & 37.75 & 38.8 & 39.82 & 40.57 & 41.77 & 43.02 \\
\hline $\begin{array}{l}\text { Depot } \\
\text { intidepressants } \ddagger\end{array}$ & 5.95 & 5.65 & 5.15 & 4.69 & 4.45 & 4.13 & 3.83 & 3.65 & 3.5 & 3.5 & 3.32 & 3.23 \\
\hline Tricyclic & 14.03 & 12.94 & 12.23 & 11.81 & 11.17 & 10.32 & 9.81 & 9.4 & 9.28 & 9.19 & 9.32 & 9.21 \\
\hline $\begin{array}{l}\text { Selective serotonin } \\
\text { reuptake inhibitors }\end{array}$ & 18.13 & 19.65 & 20.27 & 20.41 & 20.92 & 21.16 & 22.58 & 22.95 & 23.8 & 24.38 & 25.23 & 25.75 \\
\hline $\begin{array}{l}\text { Other } \\
\text { antidepressants§ }\end{array}$ & 6.87 & 8.7 & 10.05 & 11.18 & 12.21 & 12.3 & 12.67 & 13.17 & 13.31 & 13.83 & 14.5 & 14.96 \\
\hline
\end{tabular}

In instances where an individual received more than one SMI diagnostic subcategory diagnoses during the study period, the patient was assigned to the last diagnostic category received, since it was likely to be based on a greater knowledge of the individual's clinical history.

Patients were considered to be associated with a medication group if they received at least one prescription of a relevant drug within the respective year

OOrganised by chapters in British National Formulary (BNF) 67 (March 2014).

$\S N o t$ listed in BNF 67 (March 2014). 


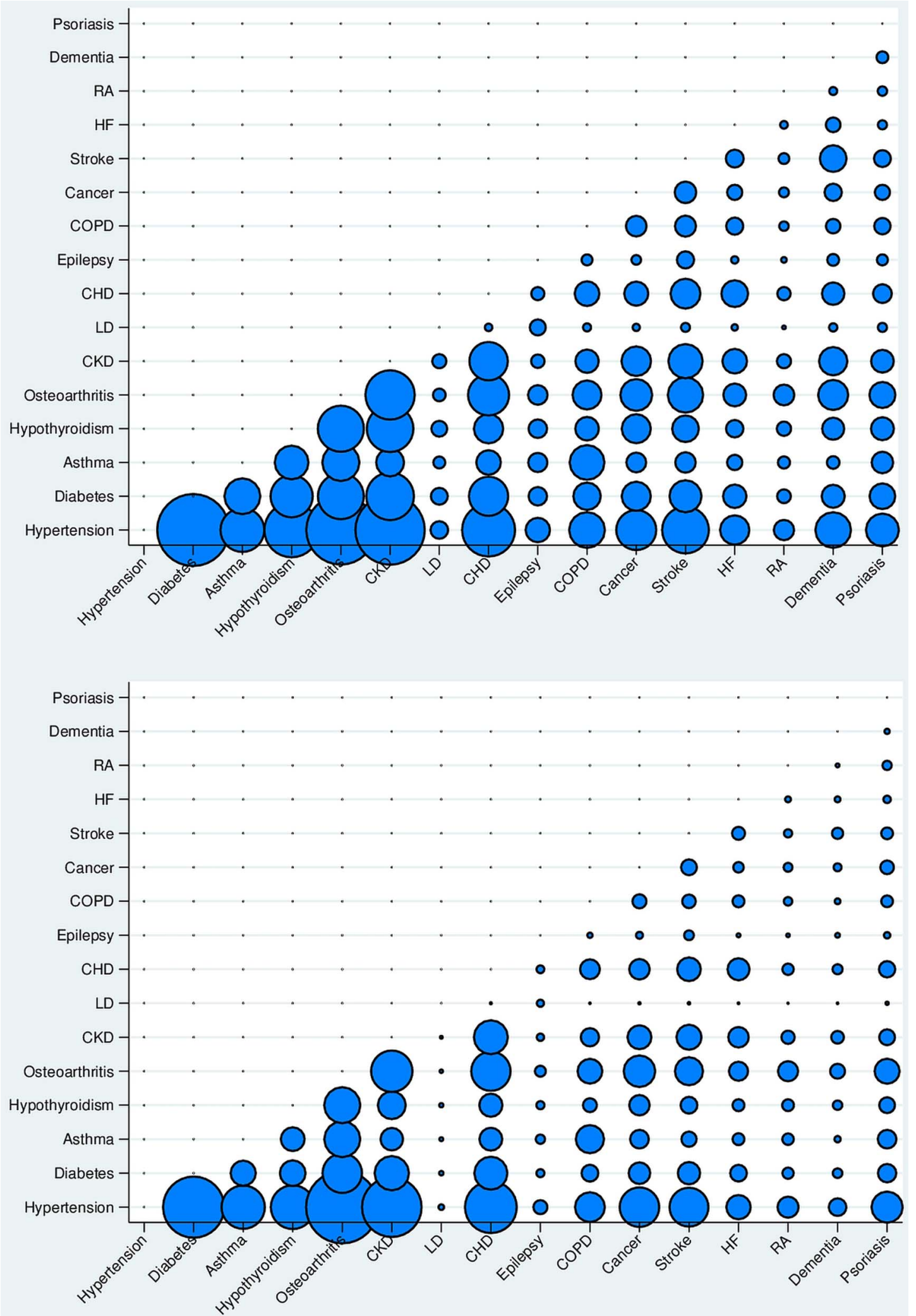

Figure 2 (top) Patterns of comorbidities for SMI (top) and control patients (bottom) over 5 years (2007/2008-2011/2012); percentage of patients with two specific conditions (see also table A3). CHD, coronary heart disease; CKD, chronic kidney disease; COPD, chronic obstructive pulmonary disease; HF, heart failure; LD, learning disability; RA, rheumatoid arthritis; SMI, severe mental illness. 
certain conditions may be made easier by the presence of an SMI diagnosis. ${ }^{40}$

The interaction between mental and physical health problems increases the costs of care which have been shown to be greater than the combined costs of having the individual conditions alone. ${ }^{41}$ Despite this, the level of mental health funding is not commensurate with burden and is lower than other chronic conditions. ${ }^{42}$ Having up to date epidemiological data helps to pinpoint how healthcare providers need to meet the challenge of providing good quality treatment and care to people with SMI.

\section{Strengths and limitations}

To our knowledge, this is the first 12-year longitudinal study to examine prevalence rates of 16 comorbidities for people with SMI compared with a matched control group without SMI. We presented these by deprivation quintile and by type of SMI diagnosis. This study demonstrates how research can use routinely collected healthcare data for this purpose. ${ }^{43}$

Databases which use routinely collected clinical data, such as the CPRD, come with certain limitations. Although the CPRD is representative of the UK in its distribution of practice location deprivation, it tends to recruit larger than average practices, ${ }^{18}$ while the version we analysed only included practices utilising one of three major clinical computer systems available (VISION). Although differences in performance across systems have been observed, ${ }^{44}$ such issues may be less relevant in this setting where we focus on prevalence rates. The main limitation, however, is that this is an observational study and the possibility of unmeasured confounding is present. Controls were matched for age, gender and practice (and since deprivation was measured at the practice level, on deprivation as well). Other important parameters such as obesity, unemployment, ethnicity, smoking status, alcohol or illegal drug use were not extracted as they may be recorded inaccurately or missing. However, the nature of the study was purely descriptive and we do not attempt to quantify associations, rather quantify overall differences in physical comorbidity irrespective of the potential underlying factors (a much stricter matching would be at risk of overcontrolling). Since we did not have specific hypotheses about the variations in prevalence rates across deprivation quintiles or between SMI and control cases, and the potential comparisons are numerous, we refrained from testing for statistically significant differences. Instead we focussed on the size of the prevalence rates and their apparent differences. Furthermore, statistical significance is less meaningful for data sets of this size. ${ }^{45}$ Second, the deprivation scores relate to the area location of the general practice and not of the individual's area of residence and therefore may be a less accurate proxy of the individual level of deprivation. ${ }^{46}$ Third, we could not quantify the severity of mental illness or of the comorbidities we investigated. Fourth, some of our estimates for specific countries and deprivation quintiles, especially for NI, are based only on a very small number of practices. Fifth, accurate identification of people with SMI requires a valid and reliable measurement of diagnosis. However, not only is there is a lack of agreement on precise diagnostic category after a diagnostic assessment, ${ }^{47}$ psychiatric diagnoses are highly variable and changeable over time and people will move between diagnoses as the nature of their illness becomes clearer (or indeed less clear). Finally, studies based on medical records will underestimate multimorbidity because some diseases are undiagnosed, and because they will not identify people who do not consult. $^{32}$

\section{Conclusions and implications for research and practice}

Our findings help our understanding of the prevalence of SMI and physical comorbidities and show rising inequalities in the pattern and number of different comorbidities and will be of interest to the scientific community, policymakers, people with SMI, their carers and professionals. Reducing these health inequalities will require adequately funded vigorous health prevention and promotion to improve the management of comorbidities with more intense action in areas of greater social and economic disadvantage. Exactly how and where this is done requires urgent attention and should be informed by the patterns of comorbidity that are most common for this group. This research provides further evidence for a number of recommendations for the NHS that have recently been made including:

- Commissioners and service providers need to be clear about the responsibilities of primary and secondary care services for monitoring and managing the physical health of people with mental health problems, starting from the beginning of treatment with identified health needs acted on quickly.

- All mental health professionals should receive basic physical health training as part of their mandatory training.

- Rates of people accessing interventions included in the QOF should be in line with predicted prevalence of the illness. 4849

The knowledge added in this study about patterns of comorbidities associated with SMI will be helpful in the development of studies aimed at investigating causal associations. Multimorbidity is a possible confounding factor, so will be important for planning intervention trials ensuring that participants with SMI are representative regarding their morbidity burden and patterns of illnesses.

\section{Author affiliations}

${ }^{1}$ Division of Health Research, Faculty of Health and Medicine, Lancaster University, Lancaster, UK

${ }^{2}$ Manchester Institute of Biotechnology, University of Manchester, Manchester, UK

${ }^{3}$ NIHR School for Primary Care Research, Centre for Primary Care, Institute of Population Health, University of Manchester, Manchester, UK 
${ }^{4}$ Department of Health Sciences, University of York, York, UK

${ }^{5}$ Centre for Biostatistics, Institute of Population Health, University of Manchester, Manchester, UK

${ }^{6}$ Centre for Pharmacoepidemiology and Drug Safety, Manchester Pharmacy School, University of Manchester, Manchester, UK

${ }^{7}$ Centre for Health Informatics, Institute of Population Health, University of Manchester, Manchester, UK

Twitter Follow Siobhan Reilly at @DrSiobhanReilly, Tim Doran at @narodmit and Evangelos Kontopantelis at @dataevan

Acknowledgements The authors would like to thank Dr Jess Drinkwater and Dr Liz England for their assistance with the categorisation of Read Codes (type of consultation and healthcare professional). They are grateful to the MHRN funded service User Researcher Panel and to Tim Rawcliffe for providing a service user review of the proposal and ongoing advice. Thanks to Jacqueline Harte for reviewing the QOF business rules and help with literature searches. They are grateful to the two reviewers for their constructive comments to the original submission. Finally, they would also like to thank our colleague, friend and collaborator, Professor Helen Lester, who sadly passed away before the publication of this paper.

Contributors SR, EK, TD, DMA, DR, CP, LG, 10 designed the study. I0 and EK extracted the data, and performed the statistical analyses. $\mathrm{CP}$ and $\mathrm{SR}$ managed the review of literature and consensus-development process for deciding and selecting the conditions to study. TD, LG and Helen Lester participated in selection of valid read codes for identifying severe mental illnesses in the CPRD. TD and HL checked the coding scheme that had been applied in a number of studies. All authors contributed to data interpretation. SR and EK led the writing of the paper with review from all authors.

Funding This work was supported by the National Institute for Health Research (NIHR) School for Primary Care Research (SPCR), under the title 'Exploring the physical health and primary care management of people with serious mental illness (SMI) using the General Practice Research Database' (project no. 144). The NIHR SPCR also supported the time of two investigators (SR and EK) through training fellowship awards during the study period. This paper presents independent research funded by the NIHR.

Disclaimer The views expressed are those of the authors and not necessarily those of the NHS, the National Institute for Health Research or the Department of Health.

\section{Competing interests None declared.}

Ethics approval This study is based on data from the Clinical Practice Research Datalink (CPRD) obtained under licence from the UK Medicines and Healthcare products Regulatory Agency. However, the interpretation and conclusions contained in this paper are those of the authors alone. The study was approved by the independent scientific advisory committee (ISAC) for CPRD research (reference number: 12_123R).

Provenance and peer review Not commissioned; externally peer reviewed.

Data sharing statement No additional data are available.

Open Access This is an Open Access article distributed in accordance with the terms of the Creative Commons Attribution (CC BY 4.0) license, which permits others to distribute, remix, adapt and build upon this work, for commercial use, provided the original work is properly cited. See: http:// creativecommons.org/licenses/by/4.0/

\section{REFERENCES}

1. Robson D, Gray R. Serious mental illness and physical health problems: a discussion paper. Int J Nurs Stud 2007;44:457-66.

2. Osborn DP, Levy G, Nazareth I, et al. Relative risk of cardiovascular and cancer mortality in people with severe mental illness from the United Kingdom's General Practice Research Database. Arch Gen Psychiatry 2007;64:242-9.

3. De Hert M, Correll CU, Bobes J, et al. Physical illness in patients with severe mental disorders. I. Prevalence, impact of medications and disparities in health care. World Psychiatry 2011;10:52-77.
4. De Hert M, Cohen D, Bobes J, et al. Physical illness in patients with severe mental disorders. II. Barriers to care, monitoring and treatment guidelines, plus recommendations at the system and individual level. World Psychiatry 2011;10:138-51.

5. Lester H. Shared care for people with mental illness: a GP's perspective. Adv Psychiatr Treat 2005;11:133-8.

6. Roberts $\mathrm{SH}$, Bailey JE. Incentives and barriers to lifestyle interventions for people with severe mental illness: a narrative synthesis of quantitative, qualitative and mixed methods studies. $J$ Adv Nurs 2011;67:690-708

7. Kontopantelis E, Springate DA, Ashcroft DM, et al. Determinants of exemption from the UK's primary care pay-for-performance programme and survival outcomes: a retrospective cohort study. BMJ Qual Saf Published Online First: 1 Dec 2015.

8. Kisely S, Smith M, Lawrence D, et al. Inequitable access for mentally ill patients to some medically necessary procedures. CMAJ 2007;176:779-84.

9. Daumit GL, Pratt LA, Crum RM, et al. Characteristics of primary care visits for individuals with severe mental illness in a national sample. Gen Hosp Psychiatry 2002;24:391-5.

10. Osby U, Brandt L, Correia N, et al. Excess mortality in bipolar and unipolar disorder in Sweden. Arch Gen Psychiatry 2001;58:844-50.

11. Brown S, Kim M, Mitchell C, et al. Twenty-five year mortality of a community cohort with schizophrenia. $\mathrm{Br} J$ Psychiatry 2010;196:116-21.

12. Barnett K, Mercer SW, Norbury M, et al. Epidemiology of multimorbidity and implications for health care, research, and medical education: a cross-sectional study. Lancet 2012;380:37-43.

13. Smith DJ, Langan J, McLean G, et al. Schizophrenia is associated with excess multiple physical-health comorbidities but low levels of recorded cardiovascular disease in primary care: cross-sectional study. BMJ Open 2013;3:pii: e002808.

14. Roberts L, Roalfe A, Wilson S, et al. Physical health care of patients with schizophrenia in primary care: a comparative study. Fam Pract 2007;24:34-40.

15. Woodhead C, Ashworth M, Schofield P, et al. Patterns of physical comulti-morbidity among patients with serious mental illness: a London borough-based cross-sectional study. BMC Fam Pract 2014;15:117

16. Osborn DPJ, Baio G, Walters K, et al. Inequalities in the provision of cardiovascular screening to people with severe mental illnesses in primary care cohort study in the United Kingdom THIN Primary Care Database 2000-2007. Schizophr Res 2011;129:104-10.

17. Janssen EM, McGinty EE, Azrin ST, et al. Review of the evidence: prevalence of medical conditions in the United States population with serious mental illness. Gen Hosp Psychiatry 2015;37:199-222.

18. Kontopantelis E, Springate D, Reeves D, et al. Withdrawing performance indicators: retrospective analysis of general practice performance under UK Quality and Outcomes Framework. BMJ 2014;348:g330.

19. NHS. Quality and outcomes framework guidance for GMS contracts 2009/10: delivering investment in general practice. http://www. nhsemployers.org/Aboutus/Publications/Documents/QOF_ Guidance 2009 final.pdf

20. Doran T, Kontopantelis E, Valderas JM, et al. Effect of financial incentives on incentivised and non-incentivised clinical activities: longitudinal analysis of data from the UK Quality and Outcomes Framework. BMJ 2011;342:d3590.

21. Springate DA, Kontopantelis E, Ashcroft DM, et al. ClinicalCodes: an online clinical codes repository to improve the validity and reproducibility of research using electronic medical records. PLOS ONE 2014;9:e99825.

22. Olier I, Springate DA, Ashcroft DM, et al. Modelling conditions and health care processes in electronic health records: an application to severe mental illness with the Clinical Practice Research Datalink. PLOSONE (in press)

23. NICE. Standards and Indicators. https://www.nice.org.uk/ standards-and-indicators

24. Communities and Local Government. The English Indices of Deprivation 2007: technical report. Government DfCaL, 2011. http:// geoconvert.mimas.ac.uk/help/imd-2007-manual.pdf

25. Sinnige J, Braspenning J, Schellevis F, et al. The prevalence of disease clusters in older adults with multiple chronic diseases-a systematic literature review. PLoS ONE 2013;8:e79641.

26. Hardoon S, Hayes JF, Blackburn R, et al. Recording of severe mental illness in united kingdom primary care, 2000-2010. PLOS ONE 2013;8:e82365

27. McGrath J, Saha S, Chant D, et al Schizophrenia: a concise overview of incidence, prevalence, and mortality. Epidemiol Rev 2008;30:67-76.

28. McEvoy JP, Meyer JM, Goff DC, et al. Prevalence of the metabolic syndrome in patients with schizophrenia: baseline 
results from the Clinical Antipsychotic Trials of Intervention Effectiveness (CATIE) schizophrenia trial and comparison with national estimates from NHANES III. Schizophr Res 2005;80: 19-32.

29. Newcomer JW. Second-generation (atypical) antipsychotics and metabolic effects: a comprehensive literature review. CNS Drugs 2005;19(Suppl 1):1-93.

30. Young SL, Taylor M, Lawrie SM. "First do no harm." A systematic review of the prevalence and management of antipsychotic adverse effects. J Psychopharmacol 2015;29:353-62.

31. Shiers DE, Rafi I, Cooper SJ, et al. Positive Cardiometabolic Health Resource: an intervention framework for patients with psychosis and schizophrenia. 2014 update. Royal College of Psychiatrists, 2014. https://www.nice.org.uk/guidance/cg155/ resources/an-intervention-framework-for-patients-with-psychosis-onantipsychotic-medication2

32. Salisbury C, Johnson L, Purdy S, et al. Epidemiology and impact of multimorbidity in primary care: a retrospective cohort study. $\mathrm{Br} J$ Gen Pract 2011;61:e12-21.

33. Kontopantelis E, Olier I, Planner C, et al. Primary care consultation rates among people with and without severe mental illness: a UK cohort study using the Clinical Practice Research Datalink. BMJ Open 2015; in press. bmjopen-2015008650.

34. Perkins AJ, Kroenke K, Unutzer J, et al. Common comorbidity scales were similar in their ability to predict health care costs and mortality. J Clin Epidemiol 2004;57:1040-8.

35. Hart JT. The inverse care law. Lancet 1971;1:405-12.

36. McLintock K, Russell AM, Alderson SL, et al. The effects of financial incentives for case finding for depression in patients with diabetes and coronary heart disease: interrupted time series analysis. $B M J$ Open 2014;4:e005178.

37. Royal College of Psychiatrists. OP88. Whole-person care: from rhetoric to reality (achieving parity between mental and physical health). Royal College of Psychiatrists, 2013:1-98
38. NHS England. Everyone counts: planning for patients 2014/15 to 2018/19. NHS England, 2013. http://www.england.nhs.uk/ wp-content/uploads/2013/12/5yr-strat-plann-guid.pdf

39. NHS England. The forward view into action: planning for 2015/16. NHS England, 2014. http://www.england.nhs.uk/wp-content/uploads/ 2014/12/forward-view-plning.pdf

40. Valderas JM, Starfield B, Sibbald B, et al. Defining comorbidity: implications for understanding health and health services. Ann Fam Med 2009;7:357-63.

41. Unutzer J, Schoenbaum M, Katon WJ, et al. Healthcare costs associated with depression in medically III fee-for-service Medicare participants. J Am Geriatr Soc 2009;57:506-10.

42. Naylor C, Parsonage M, McDaid D, et al. Long-term conditions and mental health. The costs of co-morbidities. The King's Fund, 2012:1-32. http://www.kingsfund.org.uk/sites/files/kf/field/field publication_file/long-term-conditions-mental-health-costcomorbidities-naylor-feb12.pdf

43. Haro JM. Roamer: a European roadmap for mental health research. Eur Psychiatr 2013;28.(Suppl 1):1-14. doi:10.1002/mpr.1406.

44. Kontopantelis E, Buchan I, Reeves D, et al. Relationship between quality of care and choice of clinical computing system: retrospective analysis of family practice performance under the UK's quality and outcomes framework. BMJ Open 2013;3:e003190.

45. Lin MF, Lucas HC, Shmueli G. Too big to fail: large samples and the p-value problem. Inform Syst Res 2013;24:906-17.

46. McLean G, Guthrie B, Watt G, et al. Practice postcode versus patient population: a comparison of data sources in England and Scotland. Int J Health Geogr 2008;7:37.

47. Regier DA, Narrow WE, Clarke DE, et al. DSM-5 field trials in the United States and Canada, part II: test-retest reliability of selected categorical diagnoses. Am J Psychiatry 2013;170:59-70.

48. Rethink. Lethal discrimination: why people with mental illness are dying needlessly and what needs to change. Rethink, 2013.

49. Medical Directorate NHS England. Our ambition to reduce premature mortality: a resource to support commissioners in setting a level of ambition. NHS England, 2014. 